Original article

\title{
Foreign body ingestion in Iranian children: a 4 years observational study
}

\author{
S. Amini-Ranjbar \\ Research Center for Health Care Management, Kerman, Iran
}

Received 30 August 2012, Accepted 25 September 2012.

(C) 2012, Amini-Ranjbar S.

C 2012, Russian Open Medical Journal

\begin{abstract}
Objective - Foreign body ingestion is common in children due to playing with everything. This study was done to identify type, site, and complication, as well as knowledge about the state of foreign body ingestion in children in Kerman/Iran. Methods - In this prospective study, during 4 years, 85 children less than 14 years old presented to the Emergency Department of Afzalipour Hospital in Kerman were studied. All children, regardless of their clinical symptoms, underwent total radiography (from neck to Pelvic) within the first hour of admission. In symptomatic patients, or sharp, long objects, and narcotic substances ingestion, prompt endoscopy was performed and in the case of foreign body lodging in the subglottic area, the patient was being referred to an ear, nose and throat specialist. For asymptomatic patients or far-access foreign body; lactulose, polyethylene glycol solution and high-fiber substances (for ingestion of diskette batteries, heroine and sharp objects respectively) were administered as medical treatments. Asymptomatic subjects who had ingested sharp objects or narcotic substances were hospitalized and observed, but other cases were followed out patiently (by phone call or face to face observation). Results - Mean age of subjects was 3.7 years with no significant difference between the two sexes. Mean age was 3.7 year. The most frequent ingested foreign body was diskette battery (28.2\%) followed by coin (21.2\%). The majority of subjects had no symptom (67\%). the most frequent endoscopic location (21.8\%) was subglot. Most complications occurred after battery ingestion. Endoscopic intervention was required in $31.8 \%$ that mostly in cases with ingestion of organic substances (77.8\%) and coin (61.1\%). There was a significant relationship between age and the type of foreign body $(p=0.033)$ and its location $(p=0.012)$. Medical treatment was completely successful in $68.2 \%$. There was no mortality. Conclusion - Manufacturing clockwork toys instead of operated toys, minting smaller coins and applying medical treatment in the cases that foreign body is not in the access of endoscope are recommended.
\end{abstract}

Keywords: foreign body ingestion, diskette battery, coin, Iran

Cite as Amini-Ranjbar S. Foreign body ingestion in Iranian children: a 4 years observational study. Russian Open Medical Journal $2012 ; 1: 0307$.

Correspondence to S. Amini-Ranjbar. Address: Afzalipour Hospital, Kerman, Iran. Phone: +983413222270. Email: nakhaeen@kmu.ac.ir

\section{Introduction}

Foreign body ingestion is a common problem in children [1] specially in preschool ages [2,3]. The most frequent ingested foreign body reported all over the world is coin [1, 2, 4-7]. About $25-57 \%$ of the cases had no complaint, (as most of the time history of ingestion is given by caretakers), therefore in these cases getting a precise history and physician's clinical suspicion can lead to the right diagnosis [8]. Radiography can only detect opaque objects and the diagnosis of other cases is possible through attention to the history and endoscopy. Although $90 \%$ of foreign bodies pass through gastrointestinal tract without difficulty $[2,8$, 9], various complications such as perforation, bleeding and obstruction may occur. Surgical intervention may be required in 1\% [8]. Prompt endoscopy recommended for all symptomatic (abdominal pain, chest pain, dysphagia, drooling, vomiting, hematemesis, respiratory distress) patient, ingestion of sharp or long objects, esophageal Diskette batteries, esophageal coin lodged longer than 24 hours. Endoscopic removal is recommended for gastric batteries longer than 24 -48 hours, gastric coin more than 3-4 weeks, and intestinal foreign bodies for more than five days at the same location $[2,9]$.
The type of ingested foreign body in children varies in different areas based on their different cultures $[3,4,10]$.

The present study was performed to identify the type, site and complications of foreign body in Iranian children.

\section{Material and Methods}

This prospective study was carried out from Oct 2005 to Oct 2009 on all children less than 14 years old referring to Afzalipour Hospital (the only pediatric endoscopic referral center) in Kerman/Iran due to ingestion foreign bodies.

After history and physical examamination, regardless of the passed time and clinical symptoms (abdominal pain, chest pain, dysphagia, drooling, vomiting, hematemesis, respiratory distress), all cases underwent plain films from neck to pelvis within first hour of their admission. In cases with meat lodging, lateral neck $X$ ray for better detection of bone was performed too. When coin lodged in esophagus, radiography was repeated 6 hours later and children with no symptom were observed closely for 24 hours.

Upper endoscopy was performed by the pediatric gastroenterologist with pediatric pentax endoscope. The child was being referred to an Ear, Nose and Throat specialist if the foreign 
body got impacted in subglot. Prompt endoscopy performed for: symptomatic patients, sharp, and long objects, narcotic substances ingestion, esophageal coin longer than 24 hours, esophageal battery.

If the coin lodged in stomach or intestine, the case was followed out- patiently weekly for 3-4 weeks, and parents were warned about red flags (abdominal pain, vomiting, bleeding and no passing within one week).

In cases that diskette battery was located in the stomach, radiographic evaluation was repeated 24 hours later. Otherwise, lactulose $(1 \mathrm{ml} / \mathrm{kg} / \mathrm{q} 2-4 \mathrm{~h})$ was administered to induce a loose stool and then taper interval to 6-8 hours and continued till battery passage. Meanwhile, these cases were followed either outpatiently (by phone) or in- patiently. Radiography was repeated if the battery did not pass in 2 days.

If sharp object was not in the access of endoscope, the patient was hospitalized and regardless of mild clinical symptoms (mild bleeding or abdominal pain) were given high-fiber diet such as cooked vegetables and fruit with pulp and repeat plain abdominal $X$ ray every 24 hours.

In children who had ingested heroin and other substance, and endoscopic removal was not possible, whole body irrigation with $40 \mathrm{ml} / \mathrm{kg} / \mathrm{h}$ polyethylene glycol was done in hospital.

Early surgical consultation with a pediatric surgeon was performed in sharp, long foreign bodies, Heroin ingestion and complicated cases.

Data gathering was done by using a questionnaire including variables of age, sex, complaint, physical findings, type of the foreign body, radiographic findings, medical treatment and outcome. The questionnaire was filled out by the researcher

\section{Results}

Mean age of children was 3.7 years ( 2 months to 14 years). The most common age was $1-3$ years $(n=37,43.5 \%)$. The ratio of male to female was approximately 1 ( 42 males and 43 females). Among 85 children one had Down syndrome. The majority of the cases $(n=57,67 \%)$ were asymptomatic. The most common symptom was vomiting (35\%). Radiography was detected location of foreign body $\ln 91.7 \%$.The most frequent ingested object was coin like battery $(n=24,28.2 \%)$ followed by coin and sharp objects (Table 1).

The most frequent ingested foreign body in children less than 3 years was diskette battery $(n=19,79.1 \%)$, while coin in older ages $(n=12,66.6 \%)$ (Table 1). The most frequent sharp object was safety needle ( 9 from 17) ( Table 1).

Sharp objects also were common in children younger than 3 years $(n=12,70.5 \%)$. There was a significant relationship $(p=0.033)$ between the type of ingested foreign body and age (Table 1$)$. The most frequent place (61.5\%) of foreign body at the time of referring to the Emergency Department was small intestine. The majority of children with intestinal foreign body were less than 3 years ( $n=33,68.7 \%$, Table 2). Also, most of impactions at subglot $(58.8 \%)$ were observed in children younger than 3 years (Table 2 ). There was a significant relationship between the location of foreign body and age ( $p=0.012$, Table 2). From all, $9.4 \%$ had ingested more than one or one type of objects. Diskette battery was the most frequent multi-ingested object. There was an underlying disease in 4.7\% (3 cases with esophageal stenosis and 1 case with Down syndrome).
Table1. The frequency distribution of type of ingested foreign bodies based on age

\begin{tabular}{lcccccc}
\hline \multirow{2}{*}{$\begin{array}{l}\text { Age, } \\
\text { years }\end{array}$} & $\begin{array}{c}\text { Diskette } \\
\text { battery }\end{array}$ & Coin & $\begin{array}{c}\text { Sharp } \\
\text { objects }\end{array}$ & $\begin{array}{c}\text { Organic } \\
\text { substances }\end{array}$ & Others & Total \\
\hline$<1$ & $6(37.5)$ & $0(0)$ & $5(31.3)$ & $1(6.3)$ & $4(25.0)$ & $16(100)$ \\
$1-3$ & $13(35.1)$ & $6(16.2)$ & $7(18.9)$ & $4(10.8)$ & $7(18.9)$ & $37(100)$ \\
$3-5$ & $3(23.1)$ & $4(30.8)$ & $2(15.4)$ & $1(7.7)$ & $3(23.1)$ & $13(100)$ \\
$>5$ & $2(10.5)$ & $8(42.1)$ & $3(15.8)$ & $3(15.8)$ & $3(15.8)$ & $19(100)$ \\
Total & $24(28.2)$ & $18(21.2)$ & $17(20.0)$ & $9(10.6)$ & $17(20.0)$ & $85(100)$ \\
\hline
\end{tabular}

$\mathrm{Pv}=0.032$

Sharp objects: safety needle $(n=9)$, pin $(n=2)$, thumbtack $(n=2)$, nail $(n=2)$, screw $(n=1)$ and sewing needle $(n=1)$.

Organic substances: fruit seeds $(n=3)$, dried whey $(n=2)$, heroin $(n=2)$, meat $(n=1)$ and walnut $(n=1)$.

Others: metal plaque $(n=3)$, beetle $(n=3)$, stone $(n=2)$, glass $(n=2)$, play slug $(n=1)$, pulp $(n=2)$, duster $(n=2)$, gold ring $(n=1)$ and plastic materials $(n=1)$.

Table 2. The frequency distribution of foreign body location based on age

\begin{tabular}{cccccc}
\multirow{2}{*}{ Age, years } & $\ldots \ldots \ldots$ & \multicolumn{4}{c}{ Location, $n(\%)$} \\
& Subglot & Esophagus & Stomach & Intestine & Total \\
\hline$<1$ & $1(7.1)$ & $1(7.1)$ & $0(0)$ & $12(85.7)$ & $14(100)$ \\
$1-3$ & $9(26.5)$ & $2(5.9)$ & $2(5.9)$ & $21(61.8)$ & $34(100)$ \\
$3-5$ & $4(33.3)$ & $0(0)$ & $3(25)$ & $5(41.7)$ & $12(100)$ \\
$>5$ & $3(16.7)$ & $5(27.8)$ & $0(0)$ & $10(55.6)$ & $18(100)$ \\
Total & $17(21.8)$ & $8(10.3)$ & $5(6.4)$ & $48(61.5)$ & $78(100)$ \\
\hline
\end{tabular}

$\mathrm{P}=0.012$. In 7 children who had ingested animals, pulp, plastic and glass, it was not possible to determine the location of the swallowed object.

Table 3. Medical management of children based on the location of foreign body

\begin{tabular}{lccc}
\multicolumn{1}{c}{ Foreign body } & \multicolumn{3}{c}{ Medical management } \\
\hline Diskette battery & $7(29.2)$ & $17(70.8)$ & $24(100)$ \\
Coin & $11(61.1)$ & $7(38.9)$ & $18(100)$ \\
Sharp objects & $2(11.8)$ & $15(88.2)$ & $17(100)$ \\
Organic substances & $7(77.8)$ & $2(22.2)$ & $9(100)$ \\
Others & $0(0)$ & $17(100)$ & $17(100)$ \\
Total & $27(31.8)$ & $58(68.2)$ & $85(100)$ \\
\hline
\end{tabular}

Foreign body was removed by upper endoscopy or laryngoscopy in $31.8 \%$ (Table 3). The most frequent site $(21.8 \%)$ was subglotic area (Table 2). The most frequent foreign bodies requiring endoscopic intervention were organic substances (77.8\%) and coin (61.1\%) (Table 3).

Complications such as upper and lower gastrointestinal bleeding $(n=6)$ and mediastinitis $(n=1)$ [Mediastinitis had occurred in a 3-year boy with impaction of a battery in subglot. He had developed fever and respiratory distress due to micro perforation after removal by ENT specialist. He relieved by antibiotic and other supportive therapy.] were observed in $8.5 \%$ of children and all of them had occurred following diskette battery ingestion $(n=4)$, and sharp or long objects $(n=2)$.

Medical treatment and supportive care were performed in $68.2 \%$ of cases with $100 \%$ success rate even in children with mild 
gastrointestinal bleeding or in cases that diskette battery was remained in bowels for more than one week. There was no significant relationship between medical intervention and the location of foreign body $(p=0.053)$. No surgical intervention or mortality occurred in our study.

\section{Discussion}

In the present study, no significant difference was found between the two sexes. This finding is in contrary to what have been mentioned in some other studies or text books [2,3]. It is worth to mention that in some other studies and text books nothing has been mentioned about sex differences in this regard [8-11] that can be attributed to the non-significance of sex role in foreign body ingestion.

The most common age of children in this study, like many other studies [7, 12] was 1-3 years. High prevalence of foreign body ingestion in these ages may be due to the habit of children in these ages to put every available object in their mouth.

In the present study, $68.2 \%$ of subjects were asymptomatic and while this rate is close to the rates reported in some studies (79\%) [10] it is very different with the reports of some other studies (25\%) [3], (50\%) [8] and (55\%) [4]. Difference in the type and size of ingested objects and the nature of foreign body in these studies can explain this difference.

In this study, radiography often $(77.65 \%)$ could determine the type of foreign body (opaque) . the difference between this finding and other studies in this regard (86\%) [10], (64\%) [8] and (96.04\%) [3] may be due to the different nature of the ingested objects (metal or nonmetal).

The most frequent ingested object in the present study, in contrary to other studies that it has been coin [1,2,4-7] was diskette battery (Afzalipour Hospital is the only pediatric endoscopic referral center in Kerman). This difference may be due to frequent use of toys with diskette battery in Iran. Diskette battery as the most frequent ingested foreign body in our study (28.2\%) had a significant higher rate in comparison to other studies that have reported $0.096 \%$ [3], 3.8\% [7] and $20 \%$ rates [4]. This difference can be related to different cultural viewpoints in toy manufacturing in different areas. sharp objects ingestion as the third ingested object $(20 \%)$, especially safety needle as the most frequent sharp object (9 from 17 cases), and significant differences with Philippine study (2.88\%) [3], Hong Kong study [10] (31\% for non-metal sharp objects), and or Taiwan study (9\% for safety needle) [4], all are evident of cultural differences in the availability of toys for children. Higher prevalence of ingestion safety needle among sharp objects in the present study is exactly because of frequent use of this object as an attaching instrument for sheet of blanket in our community.

The presence of significant relationship between age and type of foreign body can be attributed to the preference of specific toys in each age. For example, higher rate of playing with small toys which have diskette battery in children less than 3 years old has lead to a higher prevalence of diskette battery ingestion in these ages. While, in older ages that children are more interested in gathering money in their private money box the frequency of coin swallowing is higher.

The presence of foreign body in intestine in most cases (61.5\%) at the time of admission ( $\mathrm{X}$ ray detection) can be attributed to the either late referring (because of late awareness of parents) or good peristaltism in children.
In the present study, 31.8\% were managed with endoscopic removal and this rate is higher in comparison to the study in Taiwan (23\%) [4] and lower compared to the Philippine study (98.06\%) [3]. These differences may be due to the differences in anatomic structure of gastrointestinal tract, size and type of swallowed object or different approaches in managing these cases.

The most frequent foreign body location in the present study (21.8\%) was subglot (endoscopic detection) that differ with other studies (51.9\%) [7]. It should be mentioned that in studies the location of foreign bodies has not been reported [8-11].

The presence of significant relationship between age and foreign body location shows that in younger ages the probability of foreign body lodging is higher because $60 \%$ of the lodged cases (13 from 25 ) had seen in children less than 3 years, while this rate in children over 5 years old was 32\% ( 8 from 25), it may be due to different size of suglottic area.

In our study, higher complications occurred after the ingestion of diskette battery as the most common ingested foreign body, while endoscopy intervention was required in organic objects ingestion specially fruit seeds $(77.8 \%)$ and coin $(61.1 \%)$ due to large size.

Medical therapy for far access objects depend on presentation and complication can be an alternate management with the success rate of $100 \%$ before any surgical intervention. However, further case-control studies are required to confirm this claim.

\section{Conclusion}

Diskette battery as the most common ingested foreign body and also the most common cause of complications (bleeding and mediastinitis) and on the other hand, higher rate of endoscopy indication in organic substances and coin ingestion as well as success rate of medical therapy in far access ingested objects, it seems that prevention is the best approach for the problem of foreign body ingestion in children. Therefore, the following items are highly recommended:

i) Replacing toys that have battery with clockwork ones,

ii) Minting coins with smaller diameters,

iii) Avoiding giving fruits with seeds to children,

iv) Depend on presentation and complication, medical therapy with close observation of the patient before surgical intervention.

\section{Acknowledgments}

I Thank to Dr. Nakhaee for his helpful comments.

Conflict of interest: None

\section{Reference}

1. Shivakumar AM, Naik AS, Prashanth KB, Yogesh BS, Hongal GF. Foriegn body in upper digestive tract. Indian J Pediatric 2004; 71: 689-693 (PMID: 15345868).

2. Emmanuel $M$, Jean-Pierre $O$. Toxic and traumatic injury of the esophagus. In: Walker WA, Durie PR, Hamilton JR (editors). Pediatric Gastrointestinal Disease. 5th ed. B.C Decker, Boston, 2008: 1277-1280.

3. Marlon T, Ediberto M. Foreign body ingestion in children: A 5 Year Experience at the Philippine, General Hospital. Acta Medica Philippina 1999; 35(3-4): 25-31.

4. Change YJ. Foreign body ingestion in children. Annal of Emergency Medicine 2008; 5(4): 484 (doi:10.1016/j.annemergmed.2008.01.312). 
5. Paul RI. Foreign body ingestion in children. Pediatrics 1993; 91: 121127.

6. Yang $\mathrm{CY}$. The management of ingested foreign bodies in the upper digestive tract. Singapore Medical Journal 1991; 32: 312-315 (PMID: 1788572).

7. Adhikari $P$, Shrestha BL, Baskota DK, Sinha BK. Accidental foreign body ingestion: Analysis of 163 cases. Archives of Otorhinolaryngology 2007; 11(3): 267-270.

8. Monte CU. Foreign body ingestion in children. American Family Physician 2005; 72(2): 287-291 (PMID: 16050452).

9. Adrian J. Caustic ingestion and foreign bodies. In: Wyllie R, Hyams Js (editors). Pediatric Gastrointestestinal Disease. 3th ed. Sounders, Philadelphia, 2006: 266-271.

10. Cheng $W$, Tam PK. Foreign body ingestion in children: experience with 1265 cases. J Pediatr Surg 1999; 34: 1472-1476 (PMID: 10549750) (doi: 10.1016/S0022-3468(99)90106-9).

11. Susan E. Foreign bodies in the esophagus. In: Behrman RE, Kligman RM, Jenson HB. Nelson Textbook of pediatrics. 17th ed. Philadelphia: Sanders, 2007: 1552.

Authors:

S. Amini-Ranjbar - MD, Associate Professor of Pediatric Gastroenterology, Research Center for Health Care Management, Kerman, Iran. 